\title{
Comparative Studies on Novel Combinations of Pregabalin plus Venlafaxine with Standard Diazepam and MArketed Combination of Alprazolam plus Sertraline for Anxiety Disorders
}

\author{
Rahul P. Pol ${ }^{1}$, Atul Ramchandra Chopade ${ }^{1,2}$, Nilofar S. Naikwade ${ }^{1}$, R. J. Dias ${ }^{3}$ \\ ${ }^{1}$ Department of Pharmacology, Appasaheb Birnale College of Pharmacy, Sangli -416416. India \\ ${ }^{2}$ Department of Pharmacology, Rajarambapu College of Pharmacy, Kasegaon, 415404. India \\ ${ }^{3}$ Dept. of Pharmacy, Govt. Polytechnic Jalgaon, Maharashtra, India
}

\section{ABSTRACT}

Summary Objective- In present study, eleven novel dose combinations of Pregabalin \&amp; Venlafaxine from minimum effective dose to higher anxiolytic dose were taken for assessment of anxiolytic activity in Swiss mice.Method - Classical animal models of anxiety vise: Elevated plus-maze model and Light \&amp; Dark Exploration test Apparatus. Diazepam was used as a standard anxiolytic. In addition the combinations were also compared with a fixed dose marketed combination of Alprazolam \&amp; Sertraline (Anxit Plus).Result — The results of the study reveal that combinations of Pregabalin \&amp; Venlafaxine (serotonin norepinephrine reuptake inhibitor) have better anxiolytic efficacy, which is comparable to Diazepam and Alprazolam-Sertraline combination.Conclusion- Further clinical studies are required for getting better idea about efficacy and clinical utility of Pregabalin-Venlafaxine combination.

Keywords: Combination of pregabalin plus venlafaxine; diazepam; alprazolam; sertraline and anxiety.

\section{Introduction}

Over the course of a lifetime, $29 \%$ of the population will suffer from an anxiety disorder ${ }^{[1-4]}$. It was reported that $18 \%$ of the population have been struggling with an anxiety disorder, and $33 \%$ of these people would rate their symptoms as severe ${ }^{[1-4]}$. As such, treatment of anxiety disorders is one of the most common problems facing the practicing psychiatrist, who frequently utilizes medications as a significant part of the treatment plan ${ }^{[2-3]}$. Benzodiazepines, selective serotonin reuptake inhibitors (SSRIs), serotonin norepinephrine reuptake inhibitors (SNRIs), buspirone, and the anticonvulsants Gabapentin and Pregabalin make up the majority of the medications used to treat these disorders ${ }^{[1-4]}$. Therefore, it is important to gain as fine an understanding as possible of the proven indications of these medications as well as the dosages shown to be of benefit ${ }^{[1-4]}$.

For last 40 years, anxiety disorders have frequently been treated with benzodiazepines ${ }^{[1-4]}$. In recent years, due to unwanted adverse effects of benzodiazepines, they are replaced by SSRIs (selective serotonin reuptake inhibitors). But the major disadvantage of SSRI's is their speed of onset of efficacy ${ }^{[5-7]}$. The evidence of co morbidity of anxiety with major depressive disorders and other mental disorders is increasing day by day ${ }^{[7]}$. Thus, an unmet medical need and market opportunity exists if an anxiolytic could be developed which has a rapid onset of action but lacks the unwanted effects of existing benzodiazepines and also shows efficacy in co morbid conditions ${ }^{[1,3,7]}$.

The evolving science and our understanding of utility of Pregabalin and Venlafaxine might represent a path forward to such a goal ${ }^{[8-11]}$. Because Pregabalin proven to have early onset of action in clinical studies and acts via novel receptor, while Venlafaxine has unique dose dependent blockade of serotonin, Norepinephrine \& Dopamine which is effective in comorbid generalized anxiety disorder with depression ${ }^{[11]}$. Thus, the combination of Pregabalin and Venlafaxine might prove a better anxiolytic drug treatment than the existing ones. As Pregabalin has good Pharmacokinetic

Copyright (C) 2018 Rahul P. Pol et al.

doi: $10.24294 /$ as.v1i3.977

EnPress Publisher LLC.This work is licensed under the Creative Commons Attribution-NonCommercial 4.0 International License (CC BY-NC 4.0). http://creativecommons.org/licenses/ by/4.0/ 
pharmacodynamic profile and its clinical effectiveness in not only limited to anxiety but other disorders also, while Venlafaxine gives the advantage of its serotonin plus noradrenaline reuptake inhibiting property ${ }^{[5-11]}$. Hence taking these encouraging facts into considerations the present study was designed for evaluation of beneficial effects of co administration of these two drugs Pregabalin and Venlafaxine.

\section{Material and methods}

\subsection{Animals used-}

Swiss albino mice, Aged between 8-10 weeks, weight between $20-25 \mathrm{~g}$, of either sex were used in the present study. Mice were housed in group of six under standard laboratory conditions of temperature and 12/12 hour light and dark cycle. They had free access to food and water. All the experiments were conducted at the time from 9.00 to 15.00 hours. Animals were deprived of food but not water 12 hour before the experiments. The animals were acclimatized to laboratory condition for not less than 10 days.

The employed experimental protocols were as per the ethical principles and guidelines and approved by institutional animal ethical committee constituted for the purpose of control and supervision of experimental animals by ministry of Environmental and Forests, Government of India, New Delhi.

\subsection{Drugs used-}

Pregabalin (Hetero Drugs Ltd), Venlafaxine (Cipla Ltd), Alprazolam (Micro Labs Ltd), Sertraline (Micro Labs Ltd), were procured as gift samples.

Diazepam of Ranbaxy laboratories was purchased from a local medical shop.

DMSO (dimethylsulphoxide), NACL (sodium chloride) of Loba chemicals, Mumbai were used in the present study.

\subsection{Selection of Doses and Administration-}

In the present study, for combination of Pregabalin \& Venlafaxine, the doses of Pregabalin \& Venlafaxine were selected as- PGL: $(3,10,30,50) \mathrm{mg} / \mathrm{kg}$ and VLF:

\section{$(2,4,8,12) \mathrm{mg} / \mathrm{kg}$}

The doses were selected on the basis of previous study. The doses ranges from minimum anxiolytic dose (e.g. $3 \mathrm{mg} / \mathrm{kg}$ for Pregabalin) to higher anxiolytic dose.

The combinations and doses of PGL \& VLF were as follows-
1] Pregabalin: $3 \mathrm{mg} / \mathrm{kg}$ + Venlafaxine: $2 \mathrm{mg} / \mathrm{kg}$ (P: $3+\mathrm{V}: 2)$;

2] Pregabalin: $3 \mathrm{mg} / \mathrm{kg}$ + Venlafaxine: $4 \mathrm{mg} / \mathrm{kg}(\mathrm{P}$ : $3+\mathrm{V}: 4)$;

3] Pregabalin: $3 \mathrm{mg} / \mathrm{kg}$ + Venlafaxine: $8 \mathrm{mg} / \mathrm{kg}(\mathrm{P}$ : $3+\mathrm{V}: 8)$;

4] Pregabalin: $3 \mathrm{mg} / \mathrm{kg}$ + Venlafaxine: $12 \mathrm{mg} / \mathrm{kg}$ (P: $3+\mathrm{V}: 12)$;

5] Pregabalin: $10 \mathrm{mg} / \mathrm{kg}$ + Venlafaxine: $2 \mathrm{mg} / \mathrm{kg}$ (P: $10+\mathrm{V}: 2)$;

6] Pregabalin: $10 \mathrm{mg} / \mathrm{kg}$ + Venlafaxine: $4 \mathrm{mg} / \mathrm{kg}$ (P: $10+\mathrm{V}: 4)$;

7] Pregabalin: $10 \mathrm{mg} / \mathrm{kg}$ + Venlafaxine: $8 \mathrm{mg} / \mathrm{kg}$ (P: $10+\mathrm{V}: 8)$;

8] Pregabalin: $30 \mathrm{mg} / \mathrm{kg}$ + Venlafaxine: $2 \mathrm{mg} / \mathrm{kg}$ (P: $30+\mathrm{V}: 2)$;

9] Pregabalin: $30 \mathrm{mg} / \mathrm{kg}$ + Venlafaxine: $4 \mathrm{mg} / \mathrm{kg}$ (P: 30+V: 4);

10] Pregabalin: $30 \mathrm{mg} / \mathrm{kg}$ + Venlafaxine: $8 \mathrm{mg} / \mathrm{kg}(\mathrm{P}$ : $30+\mathrm{V}: 8)$;

11] Pregabalin: $50 \mathrm{mg} / \mathrm{kg}+$ Venlafaxine: $2 \mathrm{mg} / \mathrm{kg}(\mathrm{P}$ : $50+\mathrm{V}: 2)$.

The doses of Alprazolam \& Sertraline in combination were selected from the marketed preparation- Anxit Plus (Alprazolam: $0.5 \mathrm{mg} / \mathrm{kg}$ \& Sertraline: $25 \mathrm{mg} / \mathrm{kg}$ ) and calculated for rodents as APZ: $0.065 \mathrm{mg} / \mathrm{kg}$ and STL: $3.25 \mathrm{mg} / \mathrm{kg}$. Diazepam $(2 \mathrm{mg} / \mathrm{kg})$ is used as a positive control. All the drugs were administered intraperitoneally.

\subsection{Assessment of anxiolytic activity using Elevated plus maze test:}

Elevated plus maze (EPM) as described by Pellow et al., 1985[12,13] consist of two open arms (37X5) and two enclosed arms (37X5X12) with $12 \mathrm{~cm}$ high wall arranged so that the arms of the same type were opposite to each other. The arms were connected with a central square with of $5 \times 5 \mathrm{~cm}$. The wooden apparatus was elevated to a height of $25 \mathrm{~cm}$. above the floor. The mice were placed individually in the center of the EPM facing towards open arm and time spent in open and enclosed arm was recorded for 5 minutes. Each animal should be used only once and the test should be carried out during a fixed time of the day (10am to $3 \mathrm{pm})$. The rationale is that the open arms are more fear-provoking and that the ratio of either time spent on open: closed arms or entries into 
open-closed arms reflect the relative "safety" of closed arms compared with the relative "fearfulness" of open arms. Anxiolytics would be expected to increase the proportion of entries into and time spent on open arms. The EPM was cleaned with hydrogen peroxide after each trial. Control group received normal saline.

\subsection{Light and dark exploration test ${ }^{[14,15]}$}

This model is based on natural aversion of mice to brightly light places. The apparatus consists of two compartments box, one dark and the other brightly light. Since anxiolytic reduces the natural aversion to light compartment. They spend more time in light compartment. Anxiogenic agents, on contrary, reduce this time and spend more time in the dark compartment. A typical apparatus consists of a wooden box $(45 \mathrm{X} 27 \mathrm{X} 27 \mathrm{~cm})$. The box is open topped and dimly illuminated (10 W white bulb). Naïve mice were placed individually in the center of the light compartment and observed for the next 5 minutes for the number of crossing between two compartments and time spent in the Light and dark compartment. Diazepam in dose of $\left(2 \mathrm{mg} / \mathrm{kgi}\right.$. .p.) was used as a reference standard ${ }^{[13]}$.

\subsection{Assessment of motor activity ${ }^{[16]}$}

In order to evaluate the possible effects on the motor co-ordination, mice were tested on the rota-rod apparatus. The apparatus consists of a bar, subdivided into three compartments by disks (Rota Rod apparatus model- K19616-2 Inco, Ambala). The bar rotated at a constant speed of $22 \mathrm{rpm}$. The animals were selected 24 $\mathrm{h}$ before by eliminating those mice that did not remain on the bar for two consecutive periods of 150-200 seconds. After the selection, animals were treated with drugs and combinations intraperitoneally or the same volume of vehicle 30 minutes before the test. The results were expressed as the time for which animals remained on the rota-rod. The cut-off time used was 150 seconds.

\subsection{Data analysis}

Data presented here was analyzed by one way analysis of variance (ANOVA) followed by Tukey's test. Data was expressed as a mean \pm S.E.M and value of $\mathrm{P}<0.05$ was considered to be statistically significant in all the cases. The statistical program used was GraphPad Prism 5.0 Version for Windows, GraphPad Software (San Diego, CA, USA).

\section{Results}

\subsection{Effects on EPM Model}

\subsubsection{Effect of individual drugs and marketed combination of APZ+STL on EPM Model-}

The effects of individual drug treatments such that Control, PGL (3 mg/kg), VLF (2 mg/kg), (APZ+STL) marketed combination, Diazepam $2 \mathrm{mg} / \mathrm{kg}$, and best (PGL+VLF) combination on the number of entries \& the time spent in an open arm of EPM test are shown in Figure 1A \& 1B. The combination P: 10+V: 8 significantly decreases anxiety in mice as indicated by increase in number of entries and time spent in open arm in EPM paradigm and the effects were comparable with the results of Diazepam and marketed (APZ+STL) combination.

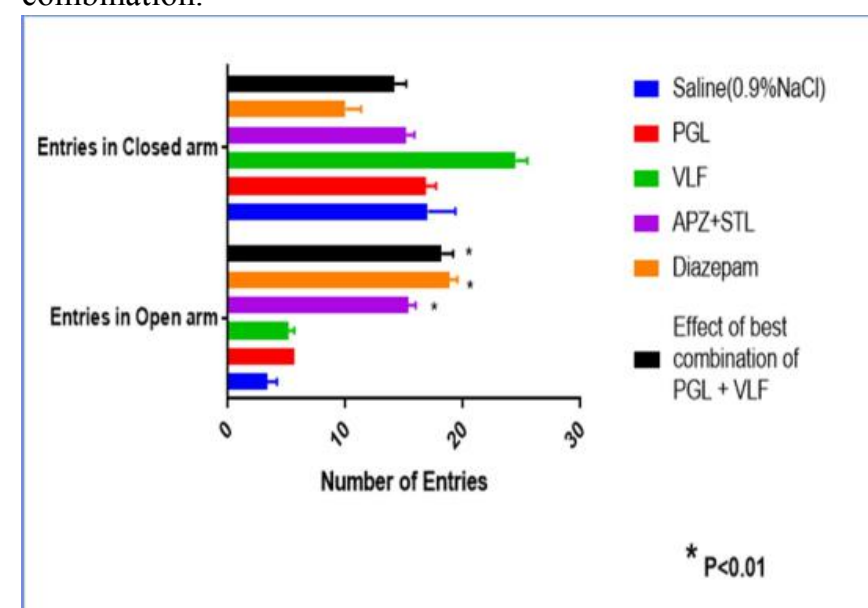

Figure 1A; Effect of individual drugs and marketed combination on number of entries in EPM Model. [Values are represented as mean $\pm \mathrm{SEM} ; \mathrm{n}=6]$.

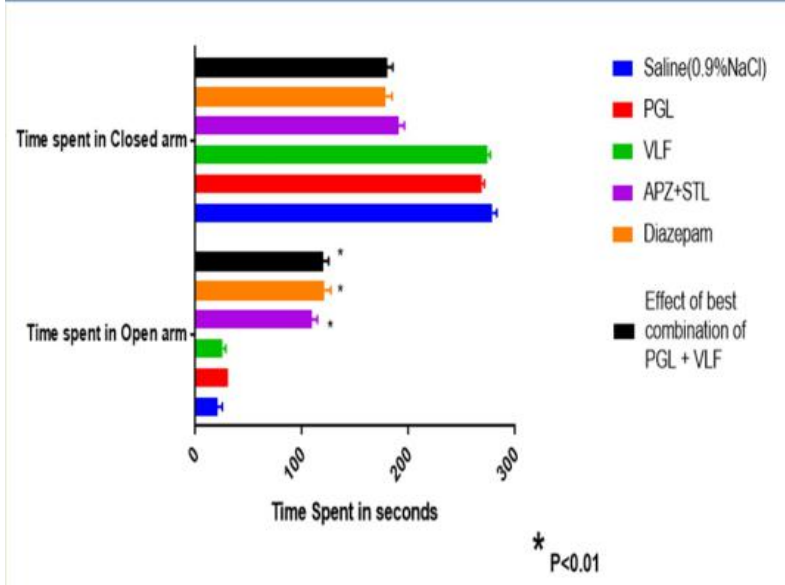

Figure 1B; Effect of individual drugs and marketed combination on time spent in both arms of EPM Model. [Values are represented as mean $\pm \mathrm{SEM} ; \mathrm{n}=6$ ]. 
Figure 1; Effect of individual drugs and marketed combination of APZ+STL on EPM Model.

3.1.2 Effect of combinations of doses of PGL \& VLF on EPM Model-

The effects of eleven combinations of doses of PGL \& VLF ie, 1] (P: 3+V: 2); 2] (P: 3+V: 4); 3] (P: 3+V: 8); 4] (P: 3+V: 12); 5] (P: 10+V: 2); 6] (P: 10+V: 4); 7] (P: 10+V: 8); 8] (P: 30+V: 2); 9] (P: 30+V: 4); 10] (P: $30+\mathrm{V}: 8)$; and 11$](\mathrm{P}: 50+\mathrm{V}: 2)$ combination on the number of entries \& the time spent in an open arm of EPM test were as per Figure No. $2 \mathrm{~A} \& 2 \mathrm{~B}$. The combination $\mathrm{P}: 10+\mathrm{V}: 8$ and combination $\mathrm{P}: 30+\mathrm{V}: 4$ significantly decreases anxiety in mice as indicated by increase in number of entries and time spent in open arm in EPM paradigm and the effects were better than any other combination.

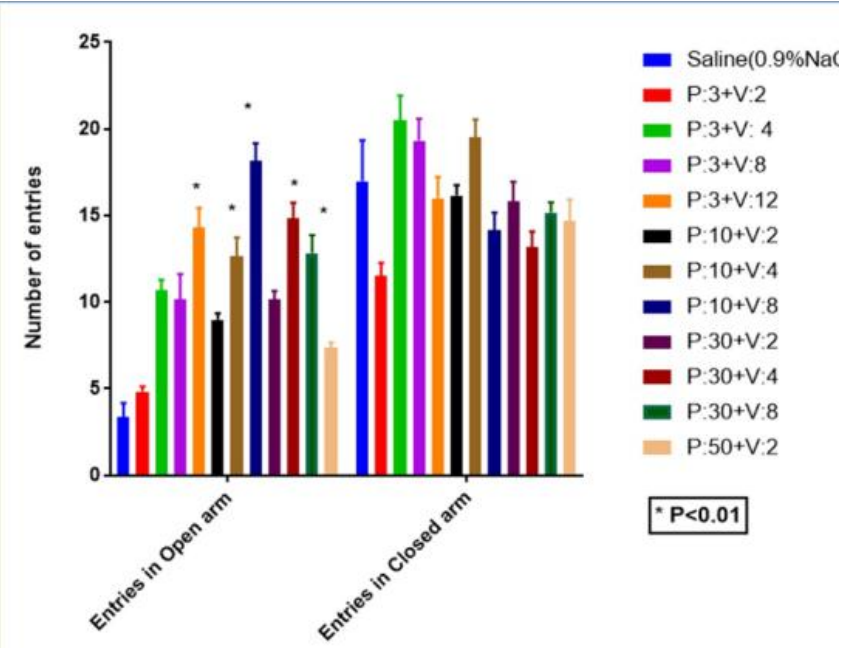

Figure 2A; Effect of combinations of doses of PGL \& VLF on number of entries in EPM Model. [Values are represented as mean $\pm S E M ; n=6]$.

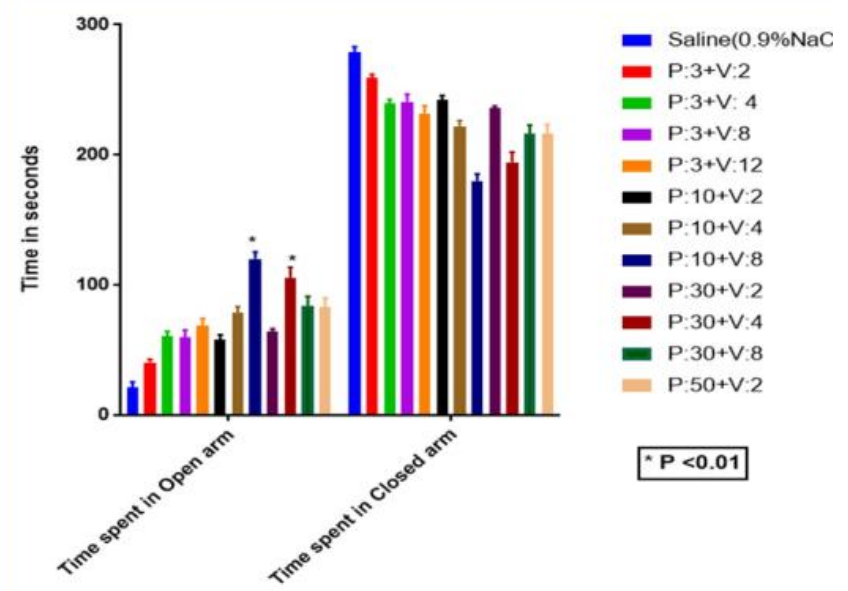

Figure 2B; Effect of combinations of doses of PGL \&
VLF on time spent in both arms of EPM Model. [Values are represented as mean $\pm \mathrm{SEM} ; \mathrm{n}=6]$.

Figure 2; Effect of combinations of doses of PGL \& VLF on EPM Model.

\subsection{Effect of L \& D Exploration Test-}

\subsubsection{Effect of individual drugs and}

marketed combination of APZ+STL on $L$ \&

\section{Exploration Test-}

The effects of individual drug treatments ie, Control, PGL ( $3 \mathrm{mg} / \mathrm{kg})$, VLF (2 mg/kg), (APZ+STL) marketed combination, Diazepam $2 \mathrm{mg} / \mathrm{kg}$, and best (PGL+VLF) combination on the number of entries $\&$ the time spent in the light compartment of L \& D Exploration Test were as per Figure No.3A \& 3B. The combination P: 10+V: 8 significantly decreases anxiety in mice as indicated by increase in number of entries and time spent in compartment of L \& D Exploration Test apparatus and the effects were comparable with the results of Diazepam and marketed (APZ+STL) combination.

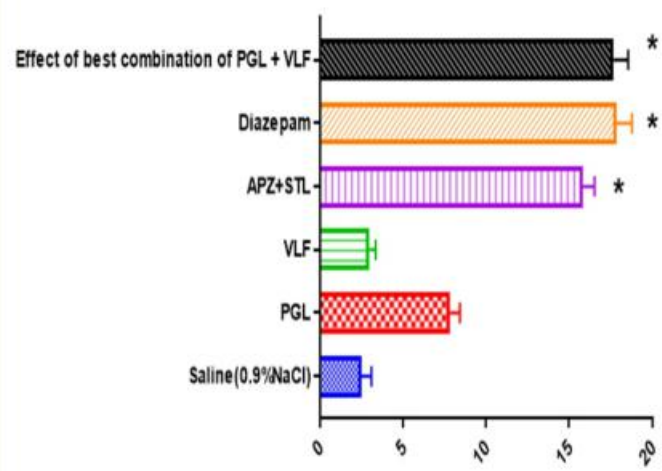

Number of Entries

Figure 3A; Effect on number of entries of individual drugs and marketed combination of APZ+STL on L \& D Exploration Test. [Values are represented as mean \pm SEM; $\mathrm{n}=6]$. 


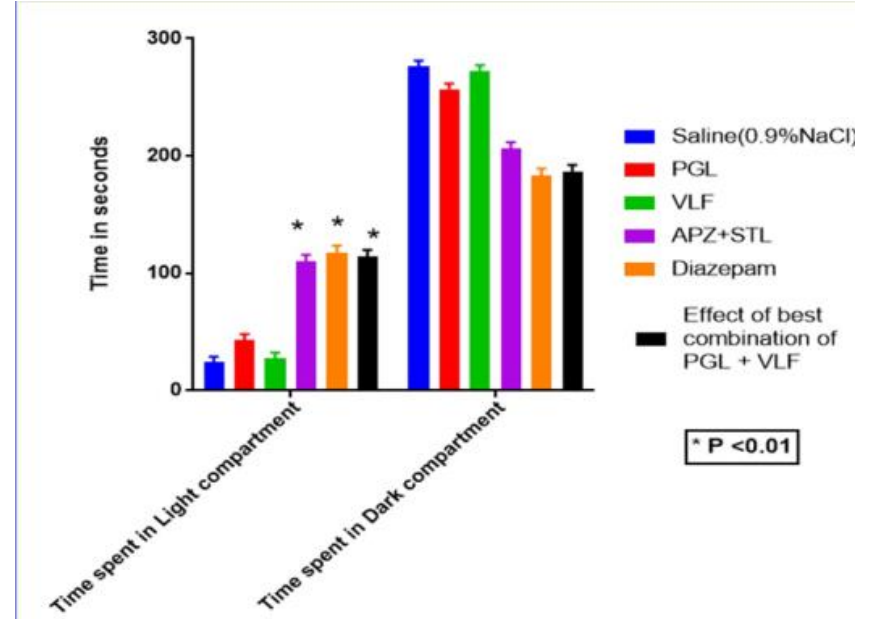

Figure 3B; Effect of individual drugs and marketed combination of APZ+STL on time spent in both compartments of L \& D Exploration Test. [Values are represented as mean \pm SEM; $\mathrm{n}=6]$.

Figure3; Effect of individual drugs and marketed combination of APZ+STL on L \& D Exploration Test. 3.2.2 Effect of combinations of doses of PGL \& VLF on L \& D Exploration Test

The effects of eleven combinations of doses of PGL \& VLF ie, 1] (P: 3+V: 2); 2] (P: 3+V: 4); 3] (P: 3+V: 8); 4] (P: 3+V: 12); 5] (P: 10+V: 2); 6] (P: 10+V: 4); 7] (P: 10+V: 8); 8] (P: 30+V: 2); 9] (P: 30+V: 4); 10] (P: 30+V: 8); and 11] (P: 50+V: 2) combination on the number of entries \& the time spent in the light compartment of L \& D Exploration Test were as per Figure No. 4A \& 4B. The combination P: $10+\mathrm{V}: 8$ and combination P: $30+\mathrm{V}$ : 4 significantly decreases anxiety in mice as indicated by increase in number of entries and time spent in the light compartment of L \& D Exploration Test apparatus and the effects were better than any other combination.

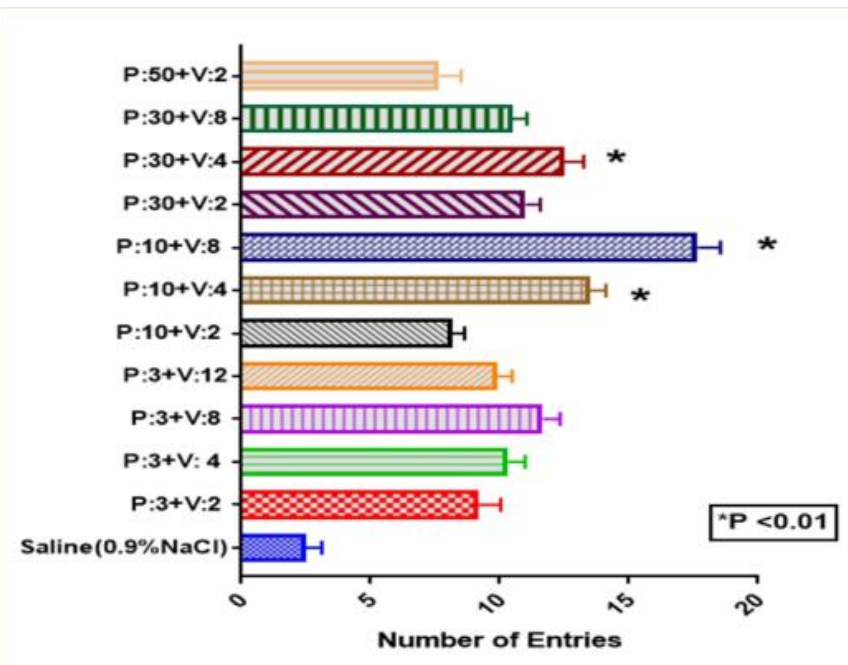

Figure 4A; Effect on number of entries of combinations of doses of PGL \& VLF on L \& D Exploration Test. [Values are represented as mean $\pm \mathrm{SEM} ; \mathrm{n}=6]$.

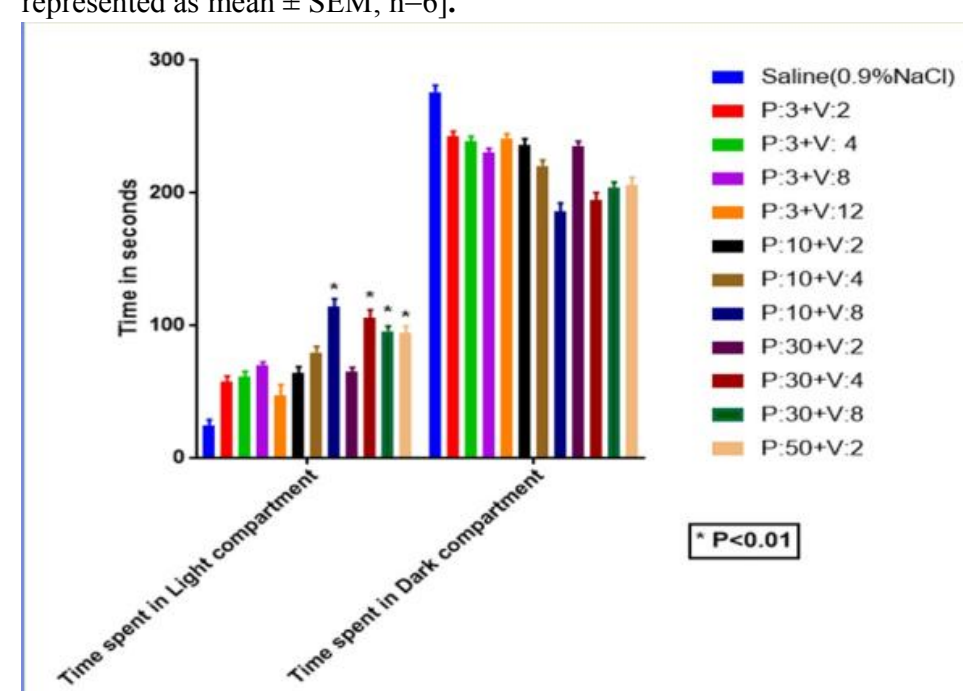

Figure 4B; Effect of combinations of doses of PGL \& VLF on time spent in both compartments of L \& D Exploration Test. [Values are represented as mean \pm SEM; $n=6$ ].

Figure 4; Effect of combinations of doses of PGL \& VLF on L \& D Exploration Test.

\subsection{Effects on motor coordination}

3.3.1 Effect of individual drugs and marketed combination of APZ+STL on motor coordination / activity using rota rod apparatus

The effects of individual drug treatments ie, Control, PGL (3 mg/kg), VLF (2 mg/kg), (APZ+STL) marketed combination, Diazepam $2 \mathrm{mg} / \mathrm{kg}$, and best (PGL+VLF) combination on the motor activity using actophotometer 
were as per Figure 5. The results of combination P: 10+V: 8 showed minimal impact on locomotor activity of mice as indicated by number of counts recorded by Actophotometer and the results were comparable with the results of Diazepam and marketed (APZ+STL) combination.

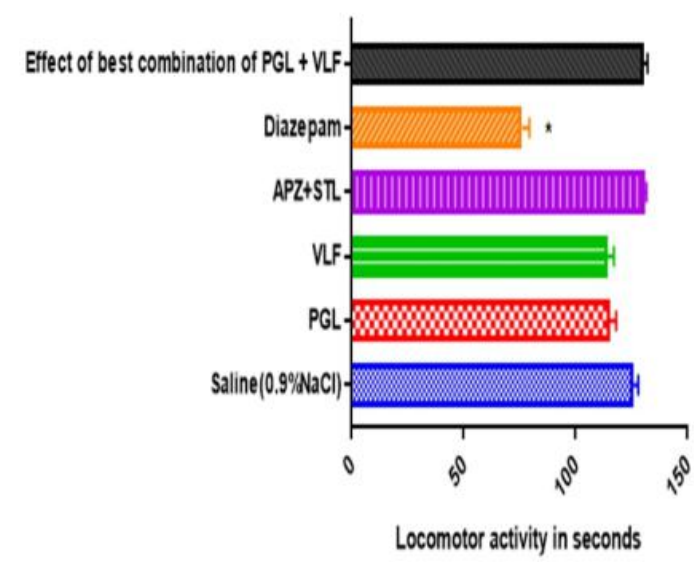

${ }^{x} \mathrm{P}<0.05$

Figure 5; Effect of individual drugs and marketed combination of APZ+STL on motor coordination/activity using rota rod apparatus. [Values are represented as mean $\pm \mathrm{SEM}$; $\mathrm{n}=6]$.

\subsubsection{Effect of combinations of doses of PGL \& VLF on motor coordination / activity using rota rod apparatus}

The effects of eleven combinations of doses of PGL \& VLF ie, 1] (P: 3+V: 2); 2] (P: 3+V: 4); 3] (P: 3+V: 8); 4] (P: 3+V: 12); 5] (P: 10+V: 2); 6] (P: 10+V: 4); 7] (P: 10+V: 8); 8] (P: 30+V: 2); 9] (P: 30+V: 4); 10] (P: $30+\mathrm{V}: 8)$; and 11] (P: 50+V: 2) combination on the number of entries \& the time spent in the light compartment of L \& D Exploration Test were as per Figure 6. The combination P: $10+\mathrm{V}: 8$ and combination P: $30+\mathrm{V}: 4$ significantly decreases anxiety in mice as indicated by increase in number of entries and time spent in the light compartment of L \& D Exploration Test apparatus and the effects were better than any other combination.

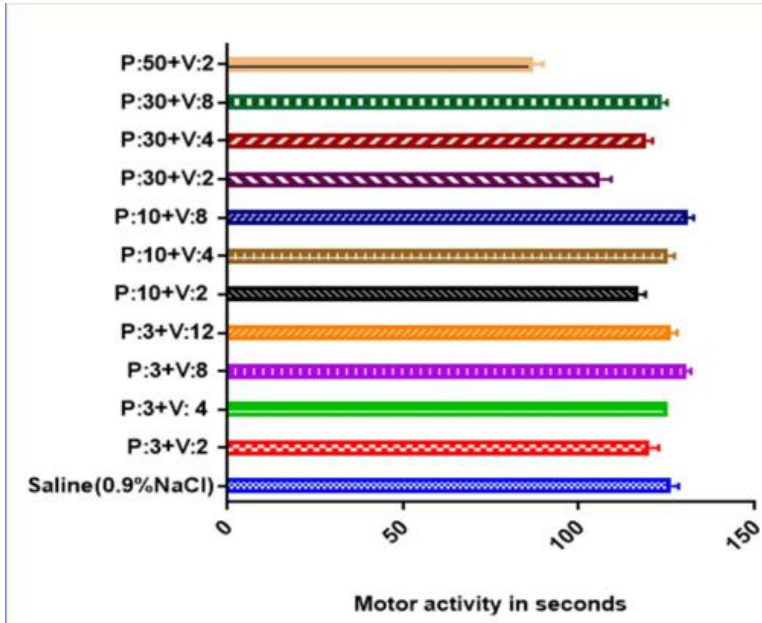

Figure 6; Effect of combinations of doses of PGL \& VLF on motor coordination/activity using rota rod apparatus. [Values are represented as mean \pm SEM; $n=6$ ].

\section{Discussion}

The present study assessed anxiolytic activity of different combinations of PGL \& VLF using two classical animal models of anxiety, the EPM model, based on the natural aversion of rodents for height and open spaces and the L\&D test, which uses the aversion of rodents for brightly lit spaces ${ }^{[13-15]}$. Diazepam was used only to compare efficacy and is not concerned with the underlying mechanism of actions of PGL \& VLF. The anxiolytic activities of PGL \& VLF are previously reported but the present study assessed efficacy of different combinations of PGL \& VLF and compared with Diazepam \& APZ-STL combination.

The result of present study of different combinations of PGL \& VLF on EPM model and L\&D E test, also considering impact on locomotor activity, revealed that, the following combinations showed effective anxiolytic profile-
a) PGL $(10 \mathrm{mg} / \mathrm{kg})+\mathrm{VLF}(4 \mathrm{mg} / \mathrm{kg})$
b) PGL $(10 \mathrm{mg} / \mathrm{kg})+\operatorname{VLF}(8 \mathrm{mg} / \mathrm{kg})$
c) PGL $(30 \mathrm{mg} / \mathrm{kg})+\mathrm{VLF}(4 \mathrm{mg} / \mathrm{kg})$
d) PGL $(30 \mathrm{mg} / \mathrm{kg})+\operatorname{VLF}(8 \mathrm{mg} / \mathrm{kg})$

Out of which, the combination PGL $(10 \mathrm{mg} / \mathrm{kg})$ + VLF $(8 \mathrm{mg} / \mathrm{kg})$ and the combination PGL $(30 \mathrm{mg} / \mathrm{kg})$ +VLF $(4 \mathrm{mg} / \mathrm{kg})$ are better because of three main reasons-

1) They inhibited locomotor activity to a lesser extent than Diazepam and thus had a better profile for anxiolytic agents.

2) Their no. of entries and time spent (s) in EPM 
and L\&D E test are almost comparable with Diazepam and APZ-STL combination.

3) Thus these combinations show almost equipotent anxiolytic efficacy with that of Diazepam $(2 \mathrm{mg} / \mathrm{kg})$ and APZ-STL combination in preclinical study on mice using classical rodent models for assessment of anxiolytic activity.

Out of these two combinations (PGL $(10 \mathrm{mg} / \mathrm{kg}$ ) $+\operatorname{VLF}(8 \mathrm{mg} / \mathrm{kg}$ ) and PGL (30mg/kg) +VLF (4mg/kg)), the combination PGL $(10 \mathrm{mg} / \mathrm{kg}) \quad+\mathrm{VLF} \quad(8 \mathrm{mg} / \mathrm{kg})$ has better results.

The non-GABAergic mechanism of PGL may have potential clinical utility in patients' refractory to benzodiazepines and also lacks retinal or optic nerve toxicity of benzodiazepines. It posses early onset of action with rapid dose adjustment, predictable drug levels \& dose response. PGL also has other advantages like- no known drug interaction, negligible metabolism (98\% renal excretion) thus no hepatic effects and easy dose adjustment in renal impairment and rapidly crosses $\mathrm{BB}^{[9-10]}$.

There is extensive preclinical and clinical evidence implicating dysfunction of serotonergic neurons in pathophysiology of anxiety ${ }^{[5]}$. In recent years, anxiety disorder has frequently been treated with SSRIs, but the major disadvantage of SSRIs is their speed of onset of efficacy ${ }^{[6]}$. VLF is superior to SSRIs in respect of its unique mechanism of action of dose dependent blockade of serotonin, norepinephrine and dopamine ${ }^{[6]}$. Today VLF XR is indicated in adults for Major Depressive Disorder, GAD, SAD and Panic Disorder. Other advantages of VLF include- no significant activity for muscarinic, histaminergic or alpha-1 adrenergic receptors in vitro and do not posses monoamine oxidase inhibitory activity $^{[6-7]}$.

Though PGL \& VLF have above advantages, they are disadvantageous in following respect-

1) PGL associated weight gain is time and dose dependent which is more likely with higher doses ${ }^{[9-10]}$.

2) During premarketing testing, seizures were reported in VLF-treated patients $(0.26 \%)$. VLF should be used cautiously in patients with history of seizures and should be promptly discontinued in any patient who develops seizures ${ }^{[3-4]}$.

But these disadvantages might be overcomes if they are given in combination, since-

1) There is increasing evidence from animal studies that 5-HT has inhibitory role in control of food intake and by inhibiting 5-HT reuptake, VLF can increase synaptic 5-HT and induce anorexia. This action serves as a treatment for obesity ${ }^{[3-4]}$.

2) PGL is basically a good anticonvulsant drug, which is more potent than gabapentine and which can easily overcome rare chances of occurrence of seizures due to VLF-treatment.

An unmet medical need and market opportunity exists if an anxiolytic could be developed which has a rapid onset of action but lacks the unwanted effects of existing benzodiazepines and also shows efficacy in co morbid conditions. The evolving science and our understanding of utility of Pregabalin and Venlafaxine might represent a path forward to such a goal. Because Pregabalin proven to have early onset of action in clinical studies and acts via novel receptor, while Venlafaxine has unique dose dependent blockade of serotonin, Norepinephrine \& Dopamine which is effective in comorbid GAD with depression ${ }^{[4-8]}$. Thus, the combination of Pregabalin and Venlafaxine might prove a better anxiolytic drug treatment than the existing ones. Hence there is need of further clinical studies of this encouraging preclinical data.

To conclude the present study revealed that two of combinations of Pregabalin and Venlafaxine, vise: Pregabalin $10 \mathrm{mg} / \mathrm{kg}+$ Venlafaxine $8 \mathrm{mg} / \mathrm{kg}$ and Pregabalin $30 \mathrm{mg} / \mathrm{kg}+$ Venlafaxine $4 \mathrm{mg} / \mathrm{kg}$ exhibited significant anxiolytic activity in mice as indicated by Elevated Plus-Maze model and Light \& Dark Exploration test without any significant influence on locomotor activity and also posses efficacy which is comparable to that of Diazepam and Alprazolam-Sertraline combination.

\section{Conflicts of interest}

We all the authors declare no conflict of interest.

\section{References}

1. Kessler RC, Berglund P, Demler O, Jin R, Merikangas KR, Walters EE. Lifetime prevalence and age-of-onset distributions of DSM-IV disorders in the National Comorbidity Survey Replication. Arch Gen Psychiatry. 2005; 62(6):593-602.

2. Davidson JR. Pharmacotherapy of social anxiety 
disorder: what does the evidence tell us? J Clin Psychiatry. 2006; 67: 20-26.

3. Davidson JR. First-line pharmacotherapy approaches for generalized anxiety disorder. J Clin Psychiatry. 2009; 70: 25-31.

4. Westenberg HG. Recent advances in understanding and treating social anxiety disorder. CNS Spectr. 2009; 14(2): 24-33.

5. Gupta YK, Prakash A. Therapeutic potential of 5-HT receptor modulators. Ind J Pharmacol. 1994; 26: 94-107.

6. Lee SI, Keltner NL. Serotonin and norepinephrine reuptake inhibitors (SNRIs): Venlafaxine and Duloxetine. Perspect. Psychi. Care. 2006.

7. Driot D, Bismuth M, Maurel A, Soulie-Albouy J, Birebent J, Oustric S, Dupouy J. Management of first depression or generalized anxiety disorder episode in adults in primary care: A systematic metareview. Presse Med. 2017; 46(12): 1124-1138.

8. Paul JW. GABA-A receptors: a viable target for novel anxiolytics? Curr Opin Pharmacol. 2006; 6: 24-29.

9. Frampton JE. Pregabalin: a review of its use in adults with generalized anxiety disorder. CNS Drugs. 2014; 28(9):835-54.

10. Parker L, Huelin R, Khankhel Z, Wasiak R, Mould J. A systematic review of pharmacoeconomic studies for pregabalin. Pain Pract. 2015; 15(1):82-94

11. Holliday SM, Benfield P. Venlafaxine. A review of its pharmacology and therapeutic potential in depression. Drugs. 1995; 49(2):280-94.

12. Pellow S, Chopin P, File SE, Briley M. Validation of open:closed arm entries in an elevated plus-maze as a measure of anxiety in the rat. J Neurosci Methods. 1985; 14: 149-167.

13. Rodgers RJ, Shepherd JK. Influence of prior maze experience on behavior and response to diazepam in the elevated plus-maze and light/ dark tests of anxiety in mice. Psychopharmacology (Berl). 1993; 113: 237-242.

14. Hascoet M. Bourin A. New approach to Light/Dark test procedure in mice. Pharmacol Biochem Behav. 1998; 60(3): 645-653.

15. Bourin M, Hascoet $M$. The mouse light/dark box test. Eur J Pharmacol. 2003; 463: 55-65.

16. Gaurav Patki, Lumeng Li, Farida Allam, Naimesh Solanki, An T Dao, Karim Alkadhi, and Samina Salim, Moderate treadmill exercise rescues anxiety and depression-like behavior as well as memory impairment in a rat model of posttraumatic stress disorder. Physiol Behav. 2014; 130: 47-53. 